\title{
Sull'irregolarità di ricezione dei segnali orari ad onde corte
}

\author{
E. Proveribio (*) \\ ricevuto il 6 novembre $\mathbf{1 9 5 9}$
}

PARIAZIONI IRREGOLARI E PERIODICHE DI INTENSTTA DI RICEZIONE.

1. La ricezione di segnali orari da parte di stazioni appositamente attrezzate permette il collegamento e l'omogeneizzazione dei vari tempi locali col Tempo Universale Uniforme Provvisorio (TU2). La rete su scala mondiale dei servizi orari dà la possibilità di confrontare non solo i vari tempi ma anche tutti i risultati e le osservazioni che contengono come parametro l'unità di tempo.

Attualmente i segnali orari tresmessi dalle stazioni trasmittenti sono di due tipi:

a) segnali di tempo ad onda portante $\left(A_{1}\right)$;

b) segnali di tempo modulati, generalmente a $1 \mathrm{KHz}\left(\mathrm{A}_{2}\right)$.

Naturalmente ogni stazione di servizio orario riceve ed utilizza segnali dei due tipi, ad onde corte, ed in misura minore ad onde lunghe.

Il problema della migliore distribuzione nel tempo dei segnali trasmessi, cosi come quello relativo alla preferenza da attribuire ai segnali a onde decametriche e metriche o a onde chilometriche e miriametriche e attualmente oggetto di seria discussione $\left({ }^{1}\right)$, soprattutto in considerazione della più elevata precisione richiesta nei confronti di segnali e di frequenze campioni, dopo l'introduzione nella tecnica della conservazione del tempo di orologi a risonanza molecolare e atomica.

D'altra parte è noto che la propagazione delle onde radioelettriche è soggetta a diversi fattori, spesso contrastanti, che ne rendono difficile lo studio e laboriosa la discussione dei risultati.

(*) Osservatorio Astronomico di Brera - Milano. 
Cosi, oltre alle difficoltà nella ricezione, derivanti dalle differenze intrinseche tra i vari segnali, numerose perturbazioni fisiche contribuiscono a rendere più difficili e incerte le misure.

Già da lungo tempo esperienze diverse hanno mostrato la dipendenza esistente tra le perturbazioni radioelettriche e i fenomeni solari, e J. H. Dellinger la potuto mettere in evidenza a questo proposito, perturbazioni periodiche con periodo eguale a quello di rotazione del Sole $\left({ }^{2}\right)$.

E noto anche, nella pratica di ricezione dei segnali orari, che esistono periodi riversi di rariabilità, legati a meccanismi e fenomeni fisici di differente natura.

Tra le principabli cause teoriche di variazioni periodiche e irregolarli sono da annoverare:

a) variazioni diurne e stagionali legate alla densità elettronica degli strati ionosferici $E(100-120 \mathrm{~km})$ e $F_{1}(220-300 \mathrm{~km})$. Come è noto, la ionizzazione di questi strati viene attribuita, in accordo soddisfacente coon l'esperienza, alla radiazione ultravioletta solare. In tal modo essa risulta funzione dell'irlaggiamento intrinseco emesso dal sole e della sua distanza zenitale:

b) variazioni diurne e irregolari, dipendenti dalla regione $F_{z}$. L'origine di queste anomalie d̀ attualmente attribuibile, oltre a radiazioni ultraviolette, all'azione di elettroni veloci, primari o secontari, di origine cromosferica. La correlazione molto evidente tra la variazione del campo magnetico terrestre e la distribuzione della zona di ionizzazione $F$. sembrerebbe avvalorare questa ipotesi;

c) perturbazioni ionosferiche a salita istantanea (PIDB), direttamente legate all'attività solare e interessanti la regione $D(80 \mathrm{~km})$, generalmente incapace di influenzare i fenomeni di propagazione ad onde corte. L'origine di questi bruschi e imponenti fenomeni di assorbimento di onde decanetriche è dovuto a anormale irraggiamento ultravioletto in dipendenza di eruzioni cromosferiche brillanti $\left({ }^{3}\right)$;

d) fenomeni di fading dovuti a interferenza di onde pereorrenti cammini leggermente diversi.

Lo studio e la interpretazione di questi fenomeni, sia dal punto di vista sperimentale che teorico, è della più grande importanza, ed ha portato un contributo fondamentale alla conoscenza della morfologia dinamica e della fisica dell'alta atmosfera ed alla fisica solare.

J)i non minore importanza sono i risultati diretti ed incliretti riguardanti il problema della propagazione delle onde radioelettriche. 
Per quanto riguarda quest'ultimo, è naturale che esso sia oggetto di grande attenzione da parte di tutti coloro che sono interessati al problema della trasmissione dei segnali orari. Per i servizi dell'ora in particolare, lo studio delle variazioni a breve e lungo periodo dell'intensità di ricezione dei segnali orari provenienti dalle piǹ diverse località può essere di grande utilità, non solo per la scelta del programma di ricezione, ma anche per lo studio dei ritardi, legati alla forma ed alla intensità degli strati ionizzati. Inoltre l'analisi e l'interpretazione di eventuali irregolarità di ricezione a breve e lungo periodo può portare qualche contributo ad una più approfondita conoscenza dei fenomeni ionosferici.

Tue ricerche effettuate da M.me $A$. Stoyko utilizzando i dati ed il materiale raccolto al B.T.H. hanno fornito recentemente importanti risultati nello studio della propagazione delle onde decametriche $\left(^{4}\right)$.

In quest'ordine di idee, sulla base del materiale raccolto in un anno e mezzo di ricezione di segnali orari al servizio dell' Ora dell' Osservatorio di Brera (VIII, 1957-II, 1959), comprendente più di 11.000 registrazioni, mi sono proposto di trovare una possibile soluzione ai seguenti due problemi di grande interesse pratico:

a) mettere in evidenza una eventuale differenza tra i vari tipi di segnali orari ricevuti:

b) individuare eventuali irregolarità periodiche o non periodiche di ricezione a lungo periodo.

\section{RISULTITI SPERIMENTIII}

2. Il metodo di riduzione dei dati utilizzati è basato sull'uso della scala di intellegibilità, applicata alla ricezione dei segnali orari. A tale scopo ciascuna stazione è stata caratterizzata, ad ogni ricezione, da un numero, corrispondente alla bontà dei segnali orari secondo la seguente scala di intelleggrbilità:

0 Assenza di segnale ndibile.

1 Segnale difficilmente intelieggibile.

2 Segnale intelleggibile solo di tanto in tanto.

3 Segnale intelleggibile.

4 Segnale bene intelleggibile.

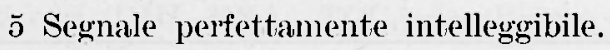


Il punteggio è stato attribuito oltre che sulla base dell'audizione auricolare in cuffia, anche utilizzando le indicazioni di un milliamperometro, situato nel circuito di registrazione all'uscita della bassa frequenza, avendo cura che la ricezione di ogni stazione avvenisse possibilmente in analoghe condizioni di sensibilità e potenza.

Per tutto il periodo in esame si è poi ritenuto che la sensibilità propria del ricevitore utilizzato non abbia subito sensibili variazioni. In tal modo il valore attribuito al segnale risulta proporzionale, con buona approssimazione, all'intensità del segnale stesso all'entrata del ricevitore.

Nella Tabella I sono elencati, l'indicativo e l'ora di trasmissione delle stazioni prese in considerazione in questa analisi con la loro rispettiva frequenza in $\mathrm{MHz}$, il tipo del segnale, la direzione geografica e la

Tabella I

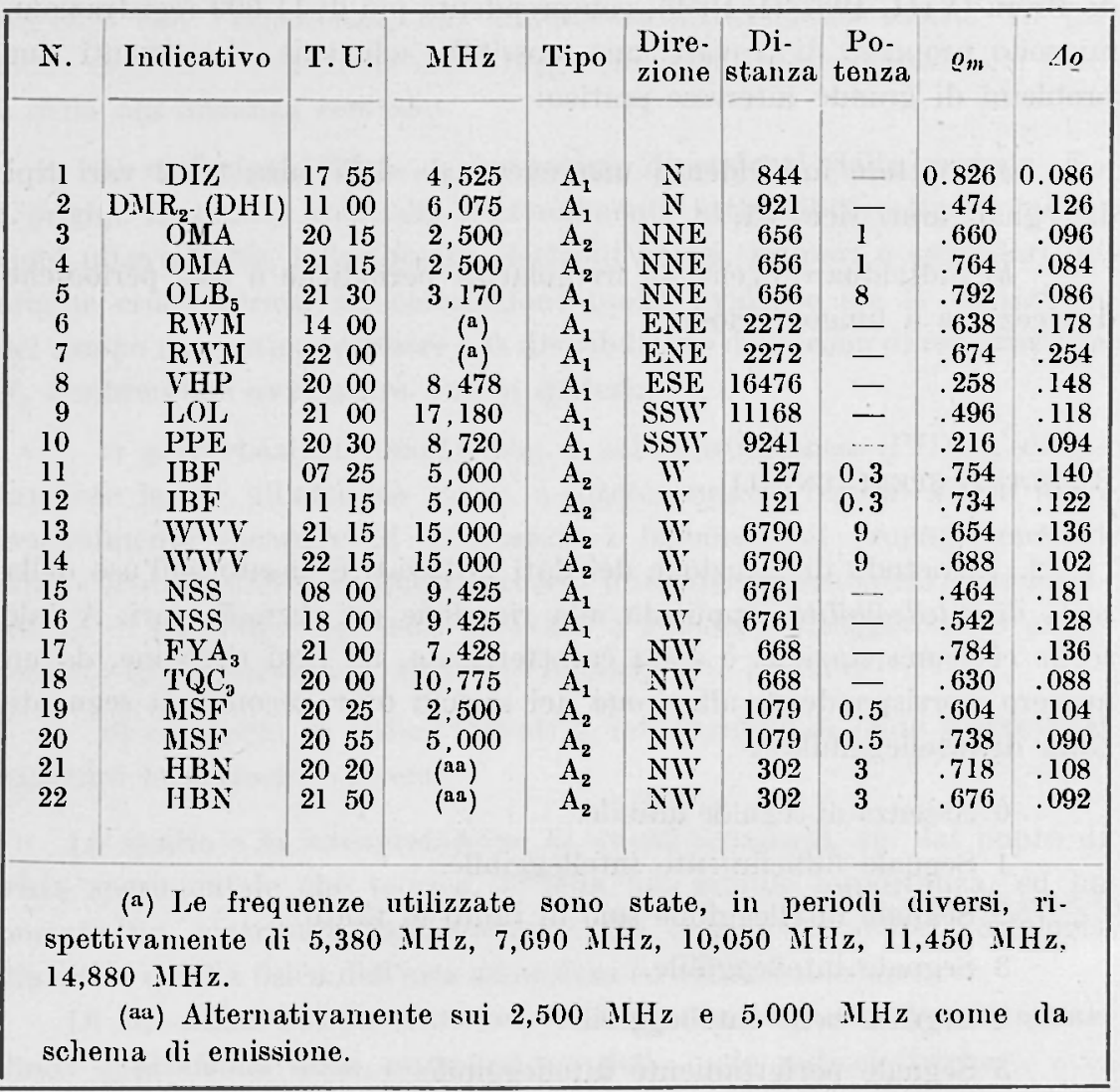


distanza in chilometri di ciascuna di esse rispetto a Milano, nonchè la potenza della portante in $\mathrm{kW}$. La figura 1 mostra invece la distribuzione delle stazioni stesse e la loro distanza relativa.

Poichè la presente ricerca ha lo scopo di mettere in evidenza eventuali periodicità o irregolarità a medio e lungo periodo, si è calcolato di quindici in quindici giorni per ogni stazione $S_{i}(i=1,2, \ldots, 22)$, il valore dell'indice di ricezione $\varrho$ per mezzo della formula

$$
\varrho=\frac{\stackrel{\sim}{ } P_{i}}{5 N}, \quad(i=1,2, \ldots, N)
$$

nella quale $P$ è il punteggio attribuito ad ogni ricezione $i$ (variabile da 0 a 5 ), ed $N$ il numpro delle ricezioni quindicinali (risultato in media eguale a 14).

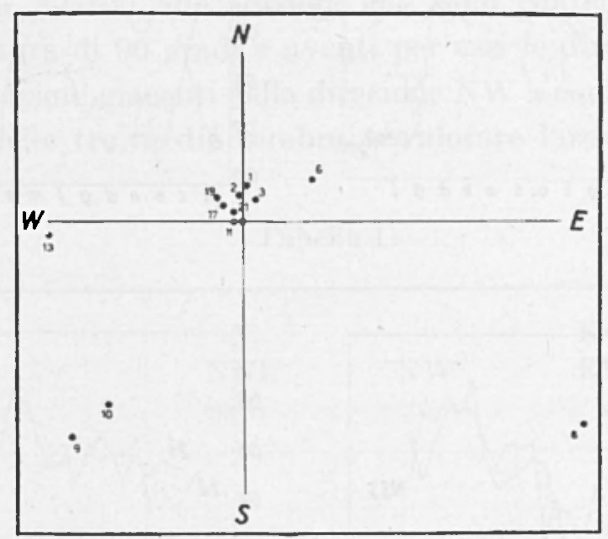

Fig. 1

I valori di o calcolati sono stati utilizzati per costruire i grafici di figura 2, che danno in funzione del tempo l'andamento di quest'ultima grandezza. I valori limiti di $\varrho=0$, e $\varrho=1$ equivalgono a ricezione nulla (segnali non udibili) ed a ricezione ottima (segnali perfettamente intelleggibili).

L'analisi di questi diagrammi permette di distinguere variazioni stagionali abbastanza evidenti (DIZ, OMA, WWV, IBF, NSS), presentanti generalmente un massimo invernale ed un minimo estivo. Contrastano con questo andamento le stazioni francesi e HBN che sembrano presentare un'inversione di fase. Sovrapposte a queste variazioni a carattere apparentemente periodico si notano variazioni irregolari, tali, in certi casi (1)HI, $\mathrm{FYA}_{3}$. $\mathrm{TQC}_{9}$ ) da alterare o nascondere le variazioni 

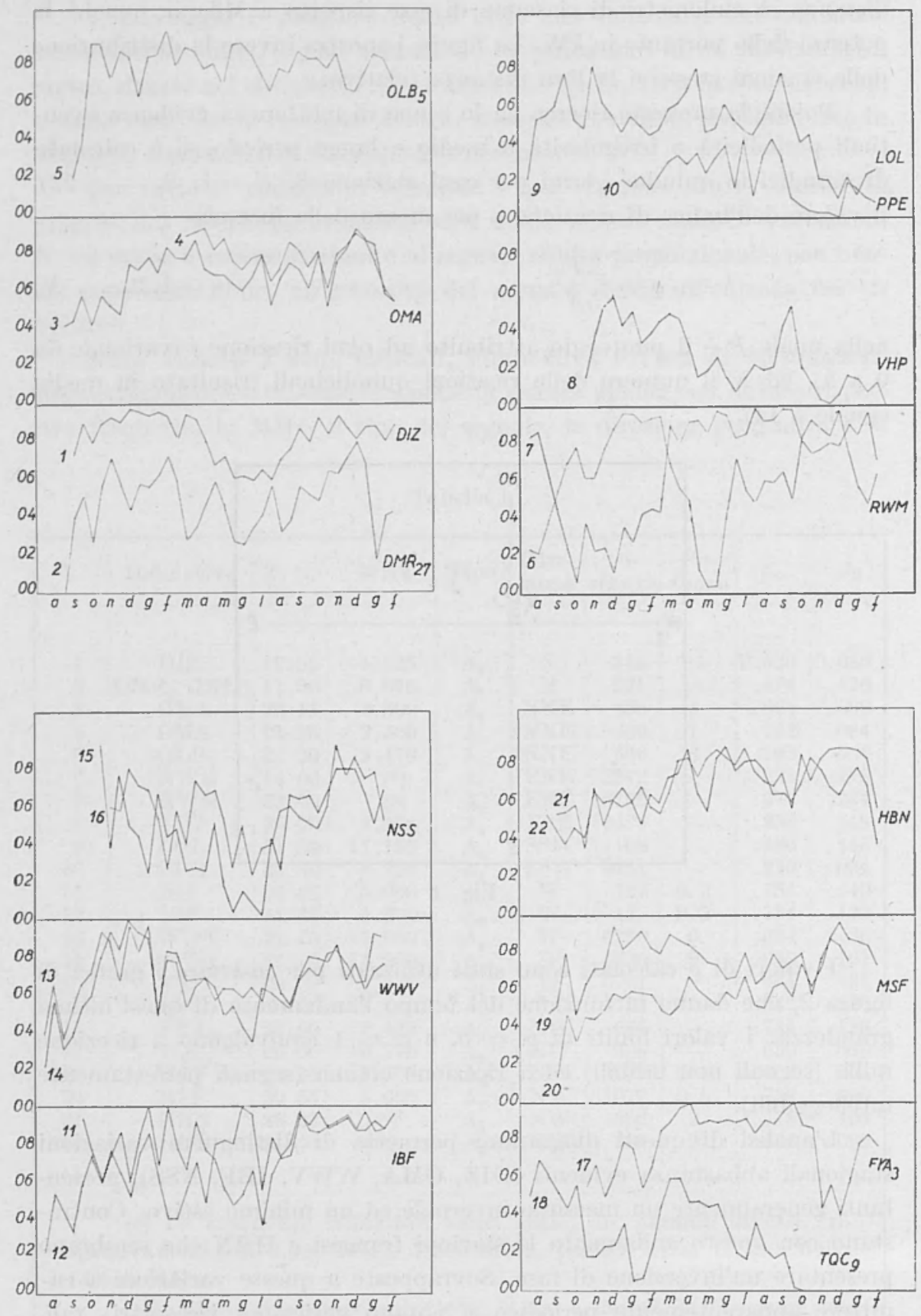

Fig. 2 
apparentemente periodiche. Di notevole interesse, ma purtroppo inficiata dalla non omogeneità delle frequenze di ricezione la stazione di Mosea (RWM).

Nelle due ultime colonne della Tabella I sono inoltre riportati a titolo indicativo i valori medi $\varrho_{m}$, calcolati graficamente, dellindice di rirezione $\varrho$ relativi a ciascun diagramma, estendentisi su un intervallo pressochè costante, con il loro scarto medio $\Delta \varrho$. L'mmpiezza di questo scarto inversamente proporzionale alla regolarità dei diagrammi stessi, da un'idea delle variazioni più o meno grandi subite dall'intensità dei segnali dumante il periodo in esame. La distribuzione dei valori di $\Delta \varrho$ presenta una interessante simmetria; questi valori infatti tendono ad aumentare andando da $\mathrm{N}$ e $\mathrm{S}$ verso $\mathrm{W}$ e $\mathrm{E}$, sempre considerando Milano come centro del sistema di orientamento. Nella Tabella II sono riportate le medie dei $\Lambda \varrho$ relativi alle stazioni che sono contenute nei due coni interi con apertura di 90 gradi e aventi per assi le direzioni NS ed EW, e quelle delle stazioni giacenti nella direzione NW a contatto dei due coni. I'andamento delle tre medie sembra avvalorare l'osservazione fatta in precedenza.

Tabella II

\begin{tabular}{|c|c|c|c|}
\hline & $\begin{array}{c}\text { N } \\
\text { NNE } \\
\text { SSW }\end{array}$ & $\mathrm{NIT}$ & $\begin{array}{c}\text { ESE } \\
\text { ENE } \\
\text { W }\end{array}$ \\
\hline Ao & 0.092 & 0.124 & 0.153 \\
\hline
\end{tabular}

Possiamo quincli sintetizzare le conclusioni che si possono trarre da questa semplice ma laboriosa analisi nei seguenti punti:

a) L'analisi dei diagrammi e in particolare dei valori calcolati dai Ao mette in evidenza una maggiore irregolarità nell'intensità di rlcezione dei segnali orari andando dalla direzione del meridiano verso quella del parallelo locali.

b) La ricezione dei segnali orari presenta in genere un andamento periodico, con apparente periodo annuo e con fase differente a seconda della stazione considerata. In generale si riscontra un massimo invernale ed un minimo estivo, o viceversa, nell'intensità di ricezione. Contempo- 
raneamente sono individuabili ampie variazioni irregrolari o con periodo minore.

Cn'ultima interessante osservazione può essere ricavata confrontando due diagrammi di una medesima stazione. relativi ad ore diverse di ricezione. Il confronto mostra una progressiva variazione di $\varrho$ in funzione dell'intervallo di tempo intercorso tra le due ricezioni (la variazione, piecola ad esempio per l'emissione di WWV a distanza di un'ora, diventa imponente per certe stazioni, come IBF, che durante il periodo estivo presenta un massimo scarto tra i due $Q$ ).

Queste conclusioni hanno spinto a cercare una possibile interpretazione sulla base della teoria della propagazione delle onde elettromagnetiche decametriche, con particolare riferimento allo studio della variazione reale od apparente dell'intensità di ricezione delle onde stesse.

PROPAGAZIONE DELLE ONDE DECAMETRICHE E INTERPRETAZIONI DELLE IRREGOLARITÀ DI RICEZIONE.

3. L'intensità trorica di propagazione delle onde decametriche dipende, come è noto, principalmente da due fattori:

a) assorbimento ionosferico in funzione della frequenza di collisione elettronica;

b) focalizzazione o diffusione delle onde elettromanetiche secondo le leggi dell'ottica geometrica della propagazione.

L'assorbimento ionosferico $\sigma=\log E_{0} / E_{1}$ cui è soggetto il campo delle onde decametriche (*) trasmesse da una stazione $A$, dovuto a cessione di energia da parte delle onde elettromagnetiche stesse agli elettroni liberi di uno strato ionosferico, è variabile da strato a strato, presentando peró un massimo di assorbimento nello strato $F\left(F_{1}\right.$ e $\left.F_{2}\right)$. I. parametri di cui $\sigma$ risulta funzione sono molto numerosi; per esempio, per lo strato $D$, responsabile di variazioni improvvise ed intense di assorbimento (PIDB), si può porre

$$
\sigma-\ldots \frac{(1+0.0035 \bar{L}) \cos ^{3 / 4} z}{\cos \alpha\left(f+f_{L}\right)^{2}}
$$

(*) $E_{0}$ rappresenta l'intensità del campo all'entrata, $E_{1}$ quella all'nscita dello strato ionosferico. 
dove 7 è un fattore di proporzionalitì, z la distanza zenitale del sole, $\alpha$ l'angolo di incidenza alla base dello strato ionizzato, e $f$ la frequenza del segnale. Poichè inoltre è

$$
R_{m}=\frac{1}{1 j} \sum_{i-m-6}^{m+6} R_{i}
$$

in cui $R$ rappresenta il numero di Wolf relativo al mese $i$, e poichè la frequenza critica $f_{L}$ risulta

$$
f^{2} L=\frac{N e^{2}}{\pi m},
$$

nella quale $e$ ed $m$ sono rispettivamente la carica e la massa dell'elettrone, ed $N$ rappresenta la densità elettronica in un punto dello strato, si può avere un'idea del numero dei fattori che influenzano l'assorbimento stesso.

Molto più complessa, sopratutto in relazione alle variazioni a lungo periodo, è l'influenza che sulla variazioni stesse di ricezione viene determinata da fenomeni di focalizzazione.

Considerando il problema in prima approssimazione, trascurando riò̀ l'azione di campi elettrici e magnetici esterni (ad esempio il campo magnetico terrestre), e considerando il problema piano (approssimazione sufficentemente accettabile fino a distanze di 1000-1500 chilometri), si ha, nell'ipotesi di una distribuzione parabolica di $N$ nello strato, che la distanza $D$ tra la stazione emittente $A$ ed il punto $A^{\prime}$ nel quale l'onda rifratta ritorna sulla Terra, può essere espressa dalla relazione

$$
D=2 h \operatorname{tang} \alpha+y \frac{f}{f_{c}} \sin \alpha \log \mid \begin{aligned}
& f_{c}+f \cos \alpha \\
& f_{c}-f \cos \alpha
\end{aligned}
$$

Questa relazione, di notevole importanza nello studio della propagazione, si presta, come è noto, a interessanti considelazioni. In essa $h$ e $y$ rappresentano rispettivamente l'altezza della base dello strato e il suo semispessore.

La distanza minima $D_{0}=2 h$ tang a rappresenta il raggio del cerchio all'interno del quale non è possibile la ricezione delle onde trasmesse dalla stazione $A$ sotto un angolo di incidenza $a$. Nella figura 3 viene presentato qualitativamente l'andamento della famiglia di curve [1], con parametro $\alpha$, in funzione di $f$. La famiglia [1] ammette l'inviluppo $I(f)$ che rappresenta la frontiera tra le coppie $(f, D)$ fisicamente compatibili e incompatibili. 
A sinistra dell'inviluppo $I(f)$ ogni punto è dunque punto d'incontro di due curve $I)\left(\alpha^{\prime}\right)==D\left(\alpha^{\prime \prime}\right)$; ciò significa che per ogni frequenza $f$ di emissione esistono due cammini diversi delle onde propagantesi in uno stesso piano ma con due diversi angoli di incidenza $a^{\prime}$, e $a^{\prime \prime}$, che pervengono in uno stesso punto alla distanzal $D(\alpha)=D\left(\alpha^{\prime \prime}\right)$ dal centro $\Lambda$ di emissione.

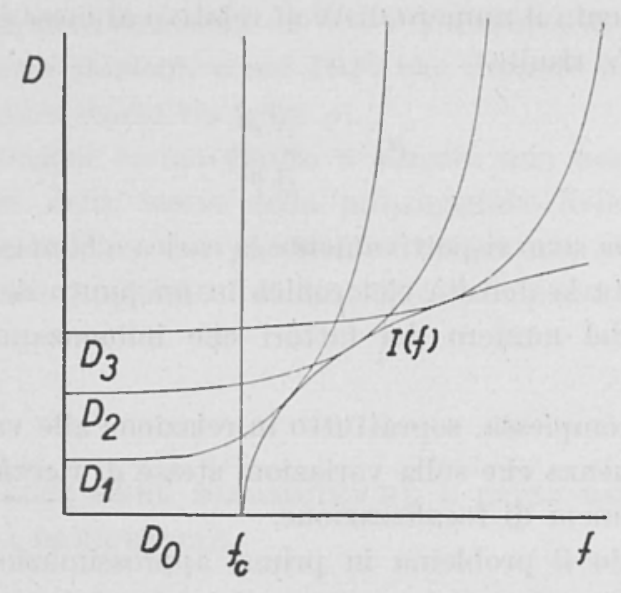

Fig. 3

Ai fini dello studio delle variazioni dell'intensità del segnale è indispensabile tener conto della distribuzione, nella zona esterna al cerchio di raggio minimo, dell'energia irradiata dalla stazione stessa. Dallo studio della funzione $D(\alpha)$, si trova che tale energia si distribuisce, nell'ipotesi semplificatrice di un'antenna non direttiva, in maniera irregolare, presentando un massimo di addensamento proprio nell'intorno del raggio $D_{0}$ (fenomeno di focalizaazione), degradando poi velocemente per distanze $\mathrm{n}>\mathrm{I}_{0}$.

Questa conclusione risulta di notevole importanza per l'interpretazione delle variazioni di intensità di ricezione, poichè essa giustifica teoricamente variazioni anche irregolari dell'intensità di ricezione in dipendenza di variazioni della frequenza $f$ di ricezione.

Ben più interessanti risultano però le conchusioni, se si tiene conto nella [1] delle variazioni dei paramentri $h, y$ e $f_{c}$, dipendenti dalle condizioni ionosferiche.

La frequenza critica $f_{c}$, oltre a variazioni dimrne, è soggetta a variazioni anmuali molto importanti, spesso indipendenti dall'attività solare, come quelle presentanti un massimo invermale ed un ninimo estivo (5). 
$A$ causa di queste variazioni si comprende come sia la distanza $I_{0}$, sia la massima frequenza $f$ utilizzabile (MUF), che puo essere direttamente ricavata da diagrammi del tipo di figura 3, subiscano oscillazioni a breve ed a lungo periodo di notevole rilievo.

Nella Tabella III sono stati calcolati i valori del laggoio $D_{0}$ della zona di silenzio per frequenze di emissione di $2,500 \mathrm{MHz}$ (valori superiori), $5,000 \mathrm{MHz}$ (valori intermedi) e $10,000 \mathrm{MHz}$ (valori inferiori), in funzione della frequenza critica $f_{c}$, espressa in $\mathrm{MHz}$, e dell'altezza virtuale $h$ di riflessione in km, utilizzando la relazione

$$
D_{0}=2 h \operatorname{tang} a=2 \pi \mid \frac{i-\frac{f_{c}^{\circ}}{f_{c}}}{.}
$$

Tabella III - Valori di $D_{\text {, }}$

\begin{tabular}{|c|c|c|c|c|c|c|}
\hline 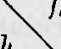 & 2 & 3 & 4 & 5 & 6 & 7 \\
\hline 300 & $\begin{array}{r}450 \\
1374 \\
2940\end{array}$ & $\begin{array}{c}0 \\
798 \\
1890\end{array}$ & $\begin{array}{c}0 \\
450 \\
13+4\end{array}$ & $\begin{array}{c}0 \\
0 \\
1038\end{array}$ & $\begin{array}{c}0 \\
0 \\
798\end{array}$ & $\begin{array}{c}0 \\
0 \\
612\end{array}$ \\
\hline 350 & $\begin{array}{r}526 \\
1604 \\
3430\end{array}$ & $\begin{array}{c}0 \\
932 \\
2204\end{array}$ & $\begin{array}{r}0 \\
525 \\
1568\end{array}$ & $\begin{array}{c}0 \\
0 \\
1212\end{array}$ & $\begin{array}{c}0 \\
0 \\
932\end{array}$ & $\begin{array}{c}0 \\
0 \\
7(04\end{array}$ \\
\hline 400 & $\begin{array}{r}600 \\
1832 \\
3920\end{array}$ & $\begin{array}{c}0 \\
1064 \\
2520\end{array}$ & $\begin{array}{c}0 \\
600 \\
1792\end{array}$ & $\begin{array}{c}0 \\
0 \\
1384\end{array}$ & $\begin{array}{r}0 \\
0 \\
1064\end{array}$ & $\begin{array}{c}0 \\
0 \\
816\end{array}$ \\
\hline
\end{tabular}

I dati di Tabellar III, messi in relazione alle variazioni a lungo e a medio periodo di $f_{c}$ e $h$, mostrano una notevolissima variabilità del laggio $D_{0}$ e una conseguente notevole variazione dell'intensità di ricezione che a questa si accompagna per effetto di focalizzazione.

4. Questa breve discussione e i risultati conseguiti, basati sull'attuale teoria della propagazione, ci permettono di attribuire le variazioni di intensità di ricezione riscontrate a fenomeni reali di assorbimento o di focalizzazione. Purtroppo la duplicità della causa e la complessità del fenomeno devono rendere cauto qualsiasi apprezzmento o deduzione affrettata. 
Solo lo studio delle variazioni stesse su un intervallo di più anni e perfezionando il metodo di misura potrebbe dare la possibilità di un'analisi più efficace dei risultati sperimentali alla luce della teoria.

La interpretazione dei diagrammi di figura 2 sulla base della teoria della propagazione delle onde decametriche può essere però indirettamente convalidata dallo studio del tempo di propagazione dei segnali orari. Infatti detto ritardo risulta funzione del cammino percorso dal segnale; e quindi, in generale, su distanze abbastanza grandi, del numero delle riflessioni che esso compie nella ionosfera. Si dovrebbe quindi avere per distanze sufficientemente elevate, e ammettendo che su tali distanze l'assorbimento possa avere in un numero sensibile di casi un effetto preponderante, un andamento parallelo tra la variayione a lungo periodo dei ritardi e quella dell'assorbimento. Di conseguenza le curve di figura 2 dovrebbero presentare, sempre per distanze convenientemente elevate, un andamento qualitativo analogo a quello ottenibile con misure di variazioni di tempo di propagazione. Gli unici dati relativi a ricerche di questo tipo sono quelli ricavati da M.me A. Stoyko al B.I.H., relativi all'emissione WWV ${ }^{6}$ ). I risultati ottenuti da M.me A. Stoyko sui ritardi di propagazione corrispondono molto bene all'andamento dei due diagrammi della stazione di Washington di figura 2, presentando un ritardo massimo estivo (massimo di assorbimento) ed un minimo invernale (minimo di assorbimento).

Sarebbe interessante a questo proposito disporre di dati relativi a stazioni diverse per poter confermare questo risultato di notevole portata per quanto riguarda lo studio dei ritardi di propagazione e la variazione a lungo periodo degli strati ionosferici.

Resta comunque viva l'esigenza, allo stato attuale della tecnica e della precisione richiesta nella trasmissione e nel confronto di segnali e di frequenze campioni, di disporre di dati osservativi, almeno approssimativi, riguardanti i parametri fondamentali che interessano il problema della propagazione $\left(f_{c}, y, h\right)$. Ciò permetterebbe di determinare, almeno in prima approssimazione, il cammino delle radionde e ricavare delle relazioni teoriche riguardanti i fenomeni di assorbimento e di propagazione da confrontare con quelli ottenuti sperimentalmente.

Lo stato insufficiente delle nostre conoscenze attuali in questo campo può però indirizzare in un prossimo futuro la tecnica della trasmissione, come abbiamo accennato all'inizio, a prendere in considerazione altri tipi di segnali, nel campo delle onde lunghe o cortissime, che presentano una propagazione molto regolare, e, sotto certe condizioni, indipendente dallo stato della ionosfera. 


\section{RIASSENTO}

Dopo aver accennato all'interesse che riveste attualmente nei servizi orari il problema della propagazione dei segnali orari ed in particolare quello relativo alle variazioni dell'intensità di ricezione ed alla sue possibili cause, si espongono $i$ risultati ottenuti sperimentalmente, relativi a queste ultime variazioni, su un periodo di circa un anno e mezzo.

Vengono quindi brevemente discusse la periodicità e le irregolarità dei diagrammi trovati e viene data una possibile interpretazione di queste sulla base della teoria della propagazione.

Il confronto delle variazioni d'intensità riscontrate con quelle relative al tempo di propagazione dei segnali orari per la stazione di WWV, studiate al B.I.H., permette di mettere in evidenza una interessante correlazione tra questi due fenomeni.

\section{$R \hat{E S U} \| \hat{E}$}

Après avoir montre l'intérêt que le problème de la propagation des signaux horaires, et en particulier celui-ci relatif à la variation de l'intensité de reception et à ses causes possibles, revêt dans les services horaires, on donne les resultats expérimentales relatifs à ces dernieres variations sur une periode d'une année et demi à peu près.

Successivement on etudie brièvement la periodicité des diagrammes trouves et on donne une probable interpretation de ceur-ci sur la base de la théorie de la propagation.

La comparaison des variations d'intensité de reception et celles relatives au temps de propagation des signaux horaires de la station de WWV, etudiées au B.I.H., permette de montrer une interessante correlation entre les deux phénomènes.

\section{ABSTRACT}

The importance of the problem of the time signal trasmissions and, particularly, of the problem of the variations of the intensity of reception and his possible causes are show. 
The results of the variations of intensity obteined experimentally during pighteen months about are examined, and a possible interpretation on the buse of the propagation's theory is given.

In the comparation of the variations of intensity with the variation of the time of travel of the rulio waves, studied at the B.I.H., a interesting comelation between this phenomena is show.

\section{BIBIJOGRAFIA}

(1) Decnux B., C. R. du Congrès Inler. de Clmonométrie, Munich, (1959).

(2) Deduincier J. IJ., Phys. Rev., ] 5 , (1935).

( ${ }^{3}$ Bureau R., TI Rapport de la Comm. pour lélude des relations entro les I'hénom. solaires et terrestres, 61, (1948).

Jovast R., TI Rapport de la ('omm. elc., I04, (1948).

(1) Sтоуко A., Anu. Fr. de Chron. 29, 62, (1959).

(5) Jounst R., L'Ionosphère, 137, Paris, 1946.

(') Storko A., 11m. Fr. de Chron., 21, (3), 193, (1951). 